\title{
Importância da realização de radiografias em posição ortostática na avaliação inicial de pacientes com fraturas toracolombares
}

\author{
Importance of performing weight-bearing radiographs in the initial \\ evaluation of patients with thoracolumbar fractures
}

Importancia de la realización de radiografías en posición ortostática en la evaluación inicial de pacientes con fracturas toraco-lumbares

\author{
Rogério Lúcio Chaves de Resende ${ }^{1}$ \\ Jefferson Soares Leal ${ }^{2}$ \\ Juliano Rodrigues dos Santos ${ }^{3}$
}

\section{RESUMO}

Objetivo: o manejo das fraturas toracolombares estáveis é controverso, especialmente em pacientes sem déficit funcional. Este estudo questiona o motivo de ser realizada uma avaliação das imagens apenas na posição supina, uma vez que, paradoxalmente, o tratamento conservador inclui o ortostatismo precoce. O propósito deste estudo é determinar se a realização de radiografias em posição ortostática influencia na definição do tratamento dos pacientes com fraturas torácicas e lombares classificadas como tipo "A" da AO-ASIF. Métodos: de janeiro de 2007 a novembro de 2008 , foram avaliados pacientes portadores de fraturas torácicas e lombares por meio de radiografias em decúbito e tomografia computadorizada, visando identificar

\section{ABSTRACT}

Objective: the management of stable thoracolumbar fractures is controversial, especially in patients without functional deficits. This study questions the reason of doing images study only in supine position since, paradoxically, nonoperative treatment includes early orthostatism. The purpose of this study is to determine the influence of weight-bearing radiographs on defining the treatment of patients with thoracolumbar fractures classified as type " $A$ " of AO-ASIF. Methods: from January 2007 to November 2008, patients with thoracolumbar fractures were evaluated by radiographs in supine position and computed tomography to identify signs of instability. Fractures classified radiographically as type

\section{RESUMEN}

Objetivo: el manejo de las fracturas toraco-lumbares estables es controvertido, especialmente en pacientes sin déficit funcional. Este estudio cuestiona el motivo por realizarse una evaluación de las imágenes apenas en la posición supina, una vez que paradoxalmente el tratamiento conservador incluye el ortatismo precoz. El propósito de este estudio es determinar si la realización de radiografias en posición ortostática influye en la definición del tratamiento de los pacientes con fracturas torácicas y lumbares, clasificadas como tipo " $A$ " de la AO-ASIF. Métodos: de enero de 2007 a noviembre de 2008 fueron evaluados pacientes portadores de fracturas torácicas y lumbares, por medio de radiografías en decúbito y

\footnotetext{
Trabalho realizado no Hospital Universitário Risoleta Tolentino Neves da Universidade Federal de Minas Gerais - UFMG - Belo Horizonte (MG), Brasil e no Hospital das Clínicas da Universidade Federal de Minas Gerais - UFMG - Belo Horizonte (MG), Brasil.

'Ortopedista; Preceptor do Grupo de Coluna Vertebral do Hospital das Clínicas da Universidade Federal de Minas Gerais - UFMG - Belo Horizonte (MG), Brasil; Hospital Universitário Risoleta Tolentino Neves da Universidade Federal de Minas Gerais - UFMG - Belo Horizonte (MG), Brasil; Biocor Instituto - Nova Lima (MG), Brasil.

${ }^{2}$ Mestre; Preceptor e Coordenador do Grupo de Coluna Vertebral do Hospital das Clínicas da Universidade Federal de Minas Gerais - UFMG - Belo Horizonte (MG), Brasil; Biocor Instituto - Nova Lima (MG), Brasil.

${ }^{3}$ Residente (R4) do Grupo de Coluna Vertebral do Hospital das Clínicas da Universidade Federal de Minas Gerais - UFMG - Belo Horizonte (MG), Brasil, e do Hospital Universitário Risoleta Tolentino Neves da Universidade Federal de Minas Gerais - UFMG - Belo Horizonte (MG), Brasil; Biocor Instituto - Nova Lima (MG), Brasil.
} 
sinais de instabilidade. As fraturas classificadas radiograficamente como tipo "A" da AO-ASIF, inicialmente por tratamento conservador, foram submetidas à realização de radiografias em ortostatismo sem o colete ortótico na fase aguda e seus parâmetros radiológicos foram reavaliados. Resultados: foram incluídos neste estudo 59 pacientes com fraturas toracolombares estáveis (T11 a L4), e todos toleraram o exame. A cifose vertebral aumentou $46,8 \%$. O colapso anterior aumentou $15,52 \%$. Três destes pacientes $(5,1 \%)$ receberam a indicação cirúrgica após radiografias em ortostatismo por apresentarem critérios de instabilidade. Conclusões: a realização das radiografias em ortostatismo mostrou-se eficiente na identificação de pacientes com instabilidade ainda não manifesta e capaz de predizer a evolução satisfatória durante o tratamento conservador, além de ser um exame diagnóstico seguro, de fácil aplicabilidade e com baixo custo. Neste estudo, um total de 5,1\% dos pacientes tiveram seu tratamento conservador alterado para o cirúrgico.
"A" of AO-ASIF, initially treated with nonoperative care, were submitted to weight-bearing radiographs without bracing in acute phase and their radiographic parameters were reevaluated. Results: 59 patients with stable thoracolumbar fractures (T11 to L4) were included in this study, and all of them tolerated the weightbearing radiographs. The vertebral kyphosis increased $46.8 \%$. The anterior collapse increased $15.52 \%$. Three of these patients (5.1\%) were submitted to surgical treatment after weight-bearing radiographs due to instability criteria. Conclusions: weight-bearing radiographs were efficient in identifying patients with instability not established yet. This exam is able to predict the satisfactory evolution during the nonoperative treatment and it is a safe and low cost diagnostic examination with easy applicability. In this study, a total of $5.1 \%$ of the patients had their treatment changed from conservative to surgical. tomografia computarizada, visando identificar señales de inestabilidad. Las fracturas clasificadas radiográficamente como tipo " $A$ " de la $A O$ $A S I F$, inicialmente de tratamiento conservador, fueron sometidas a la realización de radiografias en ortostatismo sin chaleco ortótico, en la fase aguda, y sus parámetros radiológicos fueron reevaluados. Resultados: fueron incluidos en este estudio 59 pacientes con fracturas torácicas y lumbares estables (T11 a L4). Todos los pacientes toleraron el examen. La cifosis vertebral aumentó $46.8 \%$. El colapso anterior aumentó $15.56 \%$. Tres de estos pacientes (5.1\%) recibieron la indicación quirúrgica después de las radiografías en ortostatismo, ya que criterios de inestabilidad fueron reconocidos. Conclusiones: la realización de las radiografias en ortostatismo mostró eficiencia en la identificación de pacientes con inestabilidad, aun no manifiesta, y capaz de predecir la evolución satisfactoria durante el tratamiento conservador, además de ser un examen de diagnóstico seguro, fácil aplicabilidad y bajo costo. En este estudio, un total de $5.1 \%$ tuvo un tratamiento conservador alterado para el quirúrgico.

DESCRIPTORES: Traumatismos vertebrales/radiografía; Posicionamiento del paciente; Supinación; Cifosis; Fracturas por compresión

\section{INTRODUÇÃO}

O manejo das fraturas toracolombares é um assunto controverso, especialmente em relação aos estudos propedêuticos e às opções terapêuticas disponíveis. A maioria dessas fraturas ocorre entre T10 e L4, sendo que $66 \%$ delas ocorrem sob força em compressão axial (tipo " $\mathrm{A}$ " da AOASIF $)^{1}$. Por serem as mais comuns, as fraturas em compressão dividem opiniões acerca do melhor tratamento, especialmente quando não há déficit neurológico associado² .

O estudo radiográfico é considerado o exame inicial para o diagnóstico, para a avaliação da gravidade e para a conduta terapêutica. $\mathrm{O}$ tratamento dessas fraturas baseiase nos aspectos anatômicos relacionados à estabilidade (colapso anterior do corpo vertebral e cifose) e no estado funcional (dor e exame neurológico). Na ausência de déficits neurológicos, protocolos e mensurações radiográficas objetivas do colapso do corpo vertebral e deformidade cifótica são descritas na literatura para excluir as fraturas consideradas instáveis, nas quais há lesão do complexo ligamentar posterior. Quando há uma fratura do tipo compressão ou explosão sem déficits, considera-se uma coluna instável quando há colapso vertebral maior que $50 \%$ da altura ou cifose local maior que $20^{\circ}$ a $30^{\circ}$. Porém, não há consenso sobre os valores absolutos, e as mensurações radiográficas são padronizadas nas radiografias em posição supina ${ }^{3}$.

Excluindo esses achados radiográficos, os pacientes com fraturas toracolombares estáveis são considerados 
candidatos ao tratamento conservador, no qual é incluído o ortostatismo e mobilidade precoces, com ou sem órte$\mathrm{se}^{4,5}$. Esses parâmetros radiográficos devem ser avaliados e documentados sequencialmente ${ }^{6}$. Questiona-se o motivo de ser realizado um estudo de imagens apenas na posição supina, uma vez que o tratamento conservador inclui o ortostatismo.

Apesar de o tratamento conservador de pacientes com fraturas torácicas e lombares estáveis ser considerado seguro e efetivo ${ }^{7}$, alguns dos pacientes portadores de fraturas estáveis apresentaram manutenção do quadro álgico e/ou progressão da deformidade durante ou após o tratamento ortótico, levantando a questionamentos em relação à "estabilidade" vertebral ${ }^{8}$.

O propósito deste estudo é determinar se a realização de radiografias em ortostatismo influencia na definição do tratamento dos pacientes com fraturas torácicas e lombares classificadas como tipo "A" da AO-ASIF.

\section{MÉTODOS}

De janeiro de 2007 a novembro de 2008, foram avaliados, em dois centros de atendimento terciário, pacientes consecutivos portadores de fraturas toracolombares. O protocolo estabelecido para fraturas sem comprometimento neurológico nestes serviços incluía, na avaliação inicial, radiografias em decúbito e tomografia computadorizada, visando identificar sinais de instabilidade.

O estudo radiográfico foi conduzido com base em dois sinais: colapso anterior vertebral e cifotização. O colapso anterior vertebral foi calculado pela porcentagem da média da soma da altura da parede anterior das duas vértebras adjacentes, acima e abaixo da fratura. Esse método possui maior validade e reprodutibilidade na avaliação da magnitude da falha em compressão. A cifotização foi calculada pelo ângulo de Cobb, como descrito na literatura ${ }^{9,10}$, levando-se em conta a placa terminal superior da vértebra adjacente acima da fratura e a placa terminal inferior da vértebra abaixo, considerado o melhor parâmetro de avaliação da cifose secundária à lesão ligamentar ${ }^{3}$.

Os pacientes portadores de fraturas torácicas e lombares isoladas - sem déficits neurológicos, não patológicas e classificadas radiograficamente como tipo "A" da AOASIF - foram acompanhados prospectivamente. Esses pacientes foram tratados conservadoramente com colete ortótico (do tipo órtese tóraco-lombo-sacra, OTLS) e então liberados às atividades leves diárias. Eles eram submetidos à reavaliação clínica e radiológica com 4, 8 e 12 semanas do trauma, ambulatorialmente. $\mathrm{O}$ uso da órtese era, geralmente, descontinuado progressivamente a partir da $12^{\mathrm{a}}$ semana.

Todos os pacientes incluídos nesses critérios foram submetidos, em seguida, à realização de radiografias em posição ortostática sem o colete ortótico, na fase aguda (até três semanas), o quanto tolerassem. Os parâmetros de cifose de Cobb e afundamento anterior do corpo vertebral foram novamente mensurados e comparados com os das radiografias em decúbito. Os pacientes que apresentaram sinais de instabilidade no estudo radiográfico em ortostatismo foram submetidos ao tratamento cirúrgico com fixação pedicular.

Pacientes com sinais de fratura patológica, déficits neurológicos, sinais radiológicos de instabilidade ou fraturas do tipo "B" ou "C" da AO-ASIF ao exame inicial, em decúbito, foram excluídos deste estudo.

$\mathrm{O}$ estudo estatístico foi realizado através do software SPSS (SPSS Inc., Chicago, IL). O teste pareado $t$ de Student foi aplicado para detectar diferenças significativamente estatísticas nas mensurações radiográficas em decúbito e em ortostatismo, para o intervalo de confiança (IC) de 95\%.

\section{RESULTADOS}

Foram incluídos neste estudo 59 pacientes com fraturas torácicas e lombares estáveis (T11 a L4), com média de 53,03 anos (15-81), sendo 32 (54,2\%) pacientes do sexo masculino e $27(45,8 \%)$ do sexo feminino. O nível fraturado mais comum foi L1, com 26 vértebras (44,1\%), seguido pelo nível T12, com 13 vértebras (22\%) (Tabela 1). Pela classificação da AO-ASIF, o tipo mais comum foi o tipo “A1.2", com 27 fraturas $(45,8 \%) \mathrm{e}$ "A1.1", com 19 fraturas $(32,2 \%)$.

A média do ângulo de Cobb foi de $7,67^{\circ}\left(\mathrm{IC}=-1,83^{\circ}\right.$ $\left.17,18^{\circ}\right)$ na avaliação em decúbito, passando para $11,26^{\circ}$ $\left(\mathrm{IC}=0,51^{\circ}-22,01^{\circ}\right)$ nas radiografias em ortostatismo - um aumento médio de $46,8 \%$ na cifose. O teste pareado $t$ de Student demonstrou que esse aumento foi estatisticamente significante $(\mathrm{p}<0,0001)$ para IC de $95 \%$.

O colapso anterior vertebral aumentou de 29,25\% $(\mathrm{IC}=18,29-40,21 \%)$, em média, para 33,79\% $(\mathrm{IC}=21,04-$ $46,54 \%$ ) na avaliação em ortostase - um aumento de $15,52 \%$. Essa alteração foi estatisticamente significante no teste pareado $t$ de Student $(\mathrm{p}<0,0001)$ para IC de $95 \%$ (Tabela 1).

Todos os pacientes toleraram a realização do exame em ortostatismo. Nenhum dos pacientes submetidos ao estudo radiográfico em ortostatismo apresentou alteração no status neurológico ou quaisquer outras intercorrências.

Quatro pacientes apresentaram mudança na indicação de tratamento sendo, então, tratados cirurgicamente. Três $(5,1 \%)$ desses pacientes receberam a indicação de tratamento cirúrgico após a realização da radiografia em ortostatismo na fase aguda, todos classificados inicialmente como "A1.2" AO-ASIF. Esses três pacientes foram reclassificados como portadores de lesões tipo "B" e não tipo "A", na classificação AO-ASIF, por sinais radiológicos de

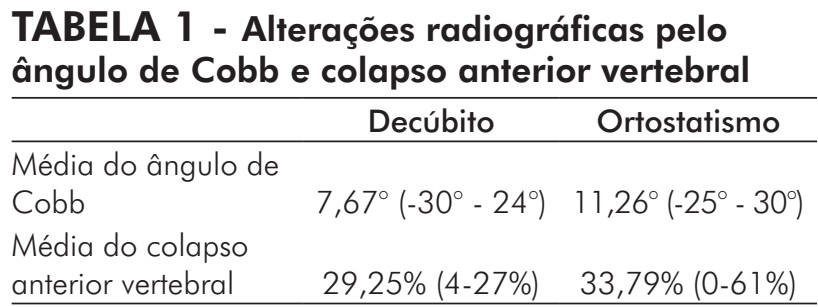


abertura posterior. M.R.P., 52 anos, sexo feminino, apresentava fratura de T12 com afundamento de $35 \%$ e cifose de Cobb de $17^{\circ}$, que aumentou para $30^{\circ}$ na radiografia em posição ortostática. I.B., 35 anos, sexo feminino, apresentava fratura de L1 com afundamento de $18 \%$ e cifose de Cobb de $10^{\circ}$. Esses parâmetros mudaram na radiografia em posição ortostática para $38 \%$ de afundamento anterior e $26^{\circ}$ de cifose de Cobb. Já C.B., 52 anos, sexo masculino, apresentava fratura de T11 com cifose de Cobb de $23^{\circ}$ e afundamento de $35 \%$, que alteraram para $44 \%$ de afundamento e $30^{\circ}$ de cifose, com claro aumento do espaço interespinhoso na visão em anteroposterior. Os autores consideraram esses três pacientes como portadores de lesão tipo "B", que foram, então, submetidos à fixação curta

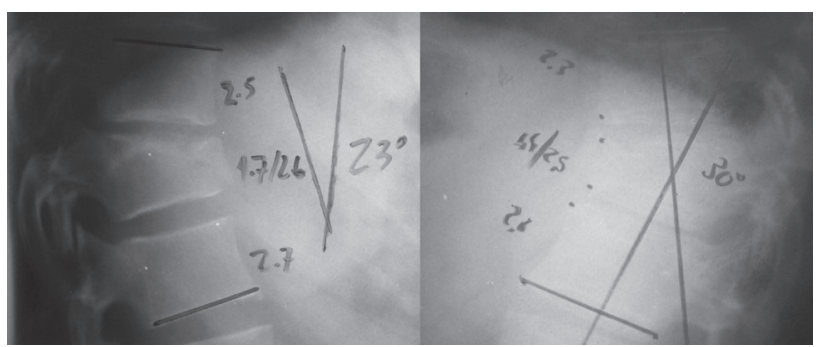

Figura 1

Paciente masculino, 52 anos, apresentando fratura de 711 , A1.2 (AO-ASIF). O ângulo de Cobb de 23, em decúbito, aumentou para $30^{\circ}$. O colapso anterior aumentou de 35 para $44 \%$.

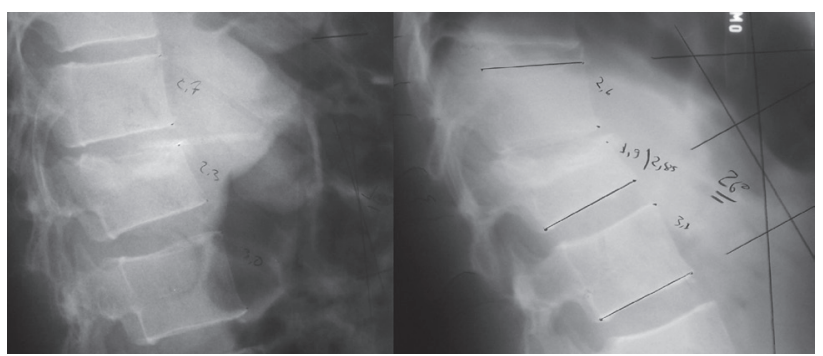

Figura 2

Paciente feminino, 35 anos, apresentando fratura de $T 17$, A3. 1 (AO-ASIF). O ângulo de Cobb de 10², em decúbito, aumentou para $26^{\circ}$. O colapso anterior aumentou de 18 para $38 \%$.

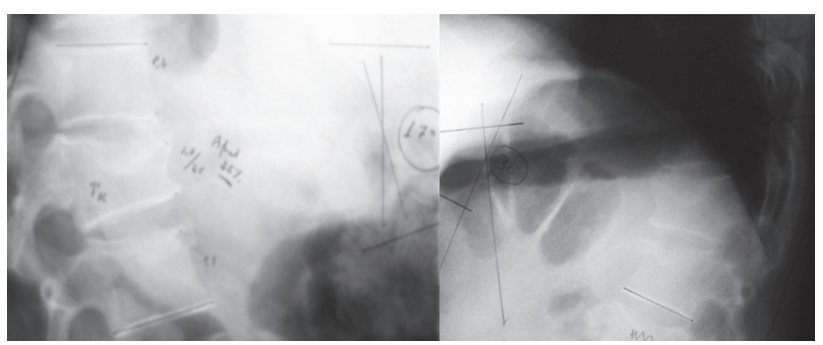

Figura 3

Paciente feminino, 52 anos, apresentando fratura de T12, A1.2 (AO-ASIF). O ângulo de Cobb de $17^{\circ}$, em decúbito, aumentou para $30^{\circ}$. O colapso anterior foi mantido em $35 \%$. com sistema pedicular, resultando em correção parcial da cifose, melhora da dor e capacidade funcional. Somente um paciente $(1,7 \%)$ teve seu tratamento conservador alterado após a fase aguda, devido à progressão do colapso anterior e da dor, sendo operado, embora ainda considerado como portador de uma fratura tipo "A" da AO-ASIF. Os demais pacientes não tiveram alterações clinicamente significativas dos seus parâmetros radiográficos durante o curso do tratamento (Figuras 1, 2 e 3).

\section{DISCUSSÃO}

A definição de tratamento em fraturas torácicas e lombares de pacientes sem déficits neurológicos é regida por parâmetros que informam sobre a estabilidade da coluna vertebral fraturada, deformidade e lesões associadas. O princípio desse tratamento é prevenir lesões neurológicas e restaurar a estabilidade vertebral, que é classicamente definida por White e Panjabi ${ }^{11}$ como a capacidade da coluna vertebral em manter suas relações anatômicas, sob cargas fisiológicas, sem lesionar a medula vertebral ou suas raízes, prevenindo a deformidade ou a dor.

Nas fraturas consideradas estáveis, o tratamento conservador com órtese permite o paciente deambular, ou seja, submeter-se a carga axial do seu próprio corpo, mas, paradoxalmente, não foram avaliados quanto à estabilidade nessa situação. A literatura, até o momento, não define com certeza como seria realizada essa avaliação e ainda apresenta resultados considerados insatisfatórios em grandes proporções ${ }^{7}$.

A indicação de tratamento cirúrgico em pacientes com fraturas sem comprometimento neurológico é regido por parâmetros anatômicos que nem sempre são considerados absolutos. Há, na literatura, vários parâmetros radiográficos que orientam o manejo dessas fraturas. Keynan et al. ${ }^{3}$ realizaram uma revisão sistemática acerca da validade e da reprodutibilidade desses métodos. Neste estudo, consideramos colapso anterior maior que $50 \%$ e cifose mensurada pelo método de Cobb maior que $25^{\circ}$ como parâmetros para indicar o tratamento cirúrgico. A extensão do colapso e a cifose, assim como a integridade da coluna posterior, são determinantes da estabilidade e podem ser considerados decisivos para o tratamento.

Petersilge et al. ${ }^{12}$ não observaram correlação entre o estudo radiográfico em decúbito das fraturas vertebrais e a integridade ligamentar posterior, principalmente por ser uma avaliação indireta e questionável. Leferink et al. ${ }^{13}$ relataram que $30 \%$ das lesões ligamentares posteriores completas podem não ser diagnosticadas por meio do estudo radiográfico e tomográfico. Particularmente, a lesão ligamentar posterior é detectada com alto índice de acurácia ( $>90 \%)$ pelo estudo de ressonância magnética, e esse achado pode indicar relativa instabilidade vertebral e consequente necessidade de tratamento cirúrgico, mas não confirma a gravidade da fratura ${ }^{14}$. Quando o exame de ressonância magnética deverá ser realizado rotineiramente ainda é motivo de debate, em especial com relação às 
fraturas em compressão sem déficits neurológicos. Alanay et al. ${ }^{15}$ acompanharam o curso natural das fraturas toracolombares do tipo explosão (tipo "A" da AO-ASIF) com integridade ligamentar posterior e revelaram que a coluna posterior intacta não é suficiente para prevenir a perda da correção da deformidade após tratamento conservador, sugerindo a importância de outros parâmetros como cominuição do corpo vertebral e placas terminais, a despeito do resultado clínico.

A realização das radiografias em ortostatismo parece ser a maneira mais prática e objetiva de avaliar se a fratura mantém parâmetros de estabilidade, sob cargas fisiológicas, inclusive sob ortostatismo.

O objetivo do tratamento é proporcionar um ambiente propício biológica e biomecanicamente à cicatrização óssea e das partes moles, com o mínimo possível de morbidade e risco. Ohana et al. ${ }^{16}$ mostraram que mesmo fratura compressiva toracolombar de $30 \%$ de acometimento do corpo vertebral pode ser tratada com a deambulação precoce e sem apoio externo. Contudo, o acompanhamento clínico e radiológico é fundamental. Acreditamos na utilização da órtese do tipo OTLS, que oferece ao paciente conforto, melhora da dor e propriocepção, sendo parte integrante do nosso protocolo de tratamento conservador. Estudos prévios mostraram que mesmo fraturas graves por explosão podem ser tratadas em regime de decúbito, redução postural e órtese ${ }^{17,18}$.

Os três $(5,1 \%)$ pacientes que receberam a indicação de tratamento cirúrgico após a realização da radiografia em ortostatismo apresentaram alterações que sugeriram instabilidade por lesão do complexo ligamentar posterior, sendo, então, reclassificados como tipo "B1.2" da AO-ASIF. Esses pacientes eram, então, portadores de lesões instáveis que não tinham sido diagnosticadas pela propedêutica usual e iriam, provavelmente, evoluir de maneira insatisfatória com o tratamento conservador. Dos pacientes que não apresentaram sinais de instabilidade na avaliação em ortostatismo, em apenas um (1,7\%) foi necessário o tratamento cirúrgico durante o curso do tratamento conservador, devido à progressão dos parâmetros radiológicos e da dor, o que sugere um alto valor preditivo negativo para progressão dos parâmetros radiográficos estudados. O tratamento cirúrgico com fixação curta pedicular estabiliza a fratura e permite mobilidade precoce. Alguns estudos mostram que, com essa técnica, mesmo sob enxertia, a recorrência da deformidade é esperada pela insuficiência da região anterior do corpo vertebral ${ }^{19-22}$.

Recentemente, Mehta et al. ${ }^{23}$ descreveram a influência da realização das radiografias em ortostatismo para o manejo das fraturas toracolombares, reforçando ainda mais o papel da lesão ligamentar posterior na determinação da estabilidade. Nesse estudo, foram incluídas apenas fraturas toracolombares de T12 a L2 (28 casos), diferentemente da nossa amostra (T11 a L4). O tempo limite para que o paciente conseguisse realizar a radiografia em ortostase foi de 48 horas. Caso contrário, ou na vigência de sinais de instabilidade, esses foram submetidos ao tratamento cirúrgico, o que limitou a chance de se tentar tratar conservadoramente alguns pacientes ainda com quadro álgico intenso. Em nosso estudo, todos os pacientes conseguiram realizar a radiografia em ortostatismo, porém, em um período maior - até três semanas do trauma - aumentando consideravelmente nossa amostra. Tal fato, associado à metodologia aplicada no nosso estudo em relação à seleção dos níveis acometidos (T11 a L4 versus T12 a L2), poderia explicar o baixo índice de instabilidade radiográfica inicialmente não diagnosticada $(5,1$ versus $25 \%)$.

\section{CONCLUSÃO}

A realização das radiografias em ortostatismo mostrou-se segura e eficiente na identificação de pacientes com instabilidade ainda não manifesta, sendo capaz de predizer a evolução satisfatória durante o tratamento conservador. Por ser um exame diagnóstico seguro, de fácil aplicabilidade e baixo custo, sugerimos estudos adicionais para confirmar a importância desse novo método de avaliação. Neste estudo, um total de 5,1\% dos pacientes teve seu tratamento conservador alterado para o cirúrgico.

\section{REFERÊNCIAS}

1. Magerl F, Aebi M, Gertzbein SD, Harms J, Nazarian S. A comprehensive classification of thoracic and lumbar injuries. Eur Spine J. 1994;3(4): 184-201.

2. Siebenga J, Leferink VJ, Segers MJ, Elzinga MJ, Bakker FC, Haarman HJ, et al. Treatment of traumatic thoracolumbar spine fractures: a multicenter prospective randomized study of operative versus nonsurgical treatment. Spine (Phila Pa 1976). 2006;31(25):2881-90.
3. Keynan O, Fisher CG, Vaccaro A, Fehlings MG, Oner FC, Dietz J, et al. Radiographic measurement parameters in thoracolumbar fractures: a systematic review and consensus statement of the spine trauma study group. Spine (Phila Pa 1976). 2006;31(5):E156-65.

4. Garfin SR. Thoracolumbar spine trauma. In: Poss R, editor. Orthopaedic Knowledge Update, vol. 3. Park Ridge, IL: American Academy of Orthopaedic Surgeons, 1990:425-40.
5. Mumford J, Weinstein JN, Spratt KF, Goel VK. Thoracolumbar burst fractures The clinical efficacy and outcome of nonoperative management. Spine (Phila Pa 1976). 1993;18(8):955-70.

6. Vaccaro AR, Kim DH, Brodke DS, Harris M, Chapman J, Schildhauer T, et al. Diagnosis and management of thoracolumbar spine fractures. J Bone Joint Surg Am. 2003;85(12):2456-70. 
7. Cantor JB, Lebwohl NH, Garvey

T, Eismont FJ. Nonoperative management of stable thoracolumbar burst fractures with early ambulation and bracing. Spine (Phila Pa 1976). 1993;18(8):971-6.

8. Keene JS, Lash EG, Kling TF Jr. Undetected posttraumatic instability of "stable" thoracolumbar fractures. J Orthop Trauma. 1988;2(3):202-11.

9. Cobb JR. The American Academy of Orthopaedic Surgeons: Instructional Course Lectures. Vol. 5. Ann Arbor, MI: JW Edwards, 1948.

10.Kuklo TR, Polly DW, Owens BD, Zeidman SM, Chang AS, Klemme WR. Measurement of thoracic and lumbar fracture kyphosis: evaluation of intraobserver, interobserver, and technique variability. Spine (Phila Pa 1976). 2001;26(1):61-5; discussion 66.

11. White AA, Panjabi MM. Clinical biomechanics of the spine, $2^{\text {nd }}$ ed. Lippincott Williams \& Wilkins, Philadelphia, 1990, p. 30-643.

12.Petersilge CA, Pathria MN, Emery SE, Masaryk TJ. Thoracolumbar burst fractures: evaluation with MR imaging. Radiology. 1995;194(1): 49-54

13.Leferink VJ, Veldhuis EF, Zimmerman $\mathrm{KW}$, ten Vergert E, ten Duis HJ. Classificational problems in ligamentary distraction type vertebral fractures: $30 \%$ of all B-type fractures are initially unrecognised. Eur Spine J. 2002;11(3):246-50.
14.Haba H, Taneichi H, Kotani Y, Terae S, Abe S, Yoshikawa H, et al. Diagnostic accuracy of magnetic resonance imaging for detecting posterior ligamentous complex injury associated with thoracic and lumbar fractures. J Neurosurg. 2003;99 (1 Suppl):20-6.

15.Alanay A, Yazici M, Acaroglu E, Turhan E, Cila A, Surat A. Course of nonsurgical management of burst fractures with intact posterior ligamentous complex: an MRI study. Spine (1 Suppl). 2004;29(21):2425-31.

16.Ohana N, Sheinis D, Rath E, Sasson A, Atar D. Is there a need for lumbar orthosis in mild compression fractures of the thoracolumbar spine?: A retrospective study comparing the radiographic results between early ambulation with and without lumbar orthosis. J Spinal Disord. 2000;13(4):305-8.

17.Rechtine GR 2nd, Cahill D, Chrin AM. Treatment of thoracolumbar trauma: comparison of complications of operative versus nonoperative treatment. J Spinal Disord. 1999;12(5):406-9.

18. Keene JS, Goletz TH, Lilleas F, Alter AJ, Sackett JF. Diagnosis of vertebral fractures. A comparison of conventional radiography, conventional tomography, and computed axial tomography. J Bone Joint Surg Am. 1982;64(4):586-94.

19.Knop C, Fabian HF, Bastian L, Blauth M. Late results of thoracolumbar fractures after posterior instrumentation and transpedicular bone grafting. Spine (Phila Pa 1976). 2001;26(1):88-99.
20.Knop C, Fabian HF, Bastian L, Rosenthal H, Lange U, Zdichavsky $\mathrm{M}$, et al. Fate of the transpedicular intervertebral bone graft after posterior stabilisation of thoracolumbar fractures. Eur Spine J. 2002;11(3): 251-7.

21.Kramer DL, Rodgers WB, Mansfield FL. Transpedicular instrumentation and short-segment fusion of thoracolumbar fractures: a prospective study using a single instrumentation system. J Orthop Trauma. 1995;9(6):499-506.

22.Leferink VJ, Zimmerman KW, Veldhuis EF, ten Vergert EM, ten Duis HJ. Thoracolumbar spinal fractures: radiological results of transpedicular fixation combined with transpedicular cancellous bone graft and posterior fusion in 183 patients. Eur Spine J. 2001;10(6):517-23.

23. Mehta JS, Reed MR, McVie JL, Sanderson PL. Weight-bearing radiographs in thoracolumbar fractures: do they influence management? Spine (Phila Pa 1976). 2004;29(5):564-7.

\section{Correspondência}

Rogério Lúcio Chaves de Resende

Rua Grão Pará, 926 - apto. 1.303 Santa Efigênia

CEP: 30140-350 - Belo Horizonte (MG), Brasil

E-mail: rogeriolucioc@yahoo.com.br 